\title{
Pregnancy in Coronavirus Disease (COVID-19) Pandemic: Clinical Opinion
}

\author{
Laila Yahya A. Alhubaishi', Atif Bashir Eltayeb Fazari1,2* \\ ${ }^{1}$ Department of Obstetrics \& Gynecology, Latifa Hospital, DHA, Dubai, UAE \\ ${ }^{2}$ Faculty of Medicine, University of Medical Sciences \& Technology, Khartoum, Sudan \\ Email: *atiffazari@hotmail.co.uk
}

How to cite this paper: Alhubaishi, L.Y.A. and Fazari, A.B.E. (2020) Pregnancy in Coronavirus Disease (COVID-19) Pandemic: Clinical Opinion. Open Journal of Obstetrics and Gynecology, 10, 708-713. https://doi.org/10.4236/ojog.2020.1050064

Received: April 20, 2020

Accepted: May 10, 2020

Published: May 13, 2020

Copyright $\odot 2020$ by author(s) and Scientific Research Publishing Inc. This work is licensed under the Creative Commons Attribution International License (CC BY 4.0).

http://creativecommons.org/licenses/by/4.0/ (c) (i) Open Access

\begin{abstract}
Coronavirus disease (COVID-19) is an infectious disease caused by a newly discovered coronavirus. This novel coronavirus is called SARS-COV-2 and the disease that it causes is called COVID-19 causes serious respiratory morbidity and mortality. We aim to spot COVID-19 disease during pregnancy during this pandemic era. COVID-19 has various clinical presentations almost same in pregnant and non-pregnant victims. The hallmark for treatment is supportive management. Vertical transmission is probable but still not well confirmed and no evidence for virus in amniotic fluid, cord blood, neonatal throat swabs, placenta swabs, genital fluid and breastmilk samples from COVID-19 infected mothers. Research is running everywhere looking step ahead for actual treatment and vaccination. COVID-19 is newly emerged disease still not well explored in many aspects looking for soon definite treatment and vaccination for prevention to roll COVID-19.
\end{abstract}

\section{Keywords}

Coronavirus-COVID-19, Pregnancy, Hydroxychloroquine, Maternal Morbidity, Maternal Mortality, Neonatal Outcome, Teratogenicity, Low Resources Countries

\section{Clinical Opinion}

Coronavirus disease (COVID-19) is an infectious disease caused by a newly discovered coronavirus. Until 2002, Coronavirus were considered minor pathogens for humans, generally associated to common cold or mild respiratory infections in immunocompetent people, with rare exceptions represented by severe infections in infants, young children and elder people. This concept completely changed with the emergence of a highly pathogenic zoonotic disease, the Severe 
Acute Respiratory Syndrome (SARS) caused by the SARS-COV. SARS mainly presented as a severe pneumonia. In late 2019, a novel coronavirus emerged in China. Since then, it has rapidly spread throughout the world and announced by World Health Organization (WHO) as pandemic disease. This novel coronavirus is called SARS-COV-2 and the disease that it causes is called COVID-19. SARS-COV2 virus causes COVID-19 disease which affects the respiratory system causing flu-like symptoms including lower and upper respiratory tract infection [1]. Coronavirus are spherical enveloped viruses with a diameter of 100 $160 \mathrm{~nm}$. Each particle contains a positive-sense single-stranded RNA genome of $27-32 \mathrm{~kb}$ that interacts with the nucleoprotein [2] [3].

SARS-COV-2 is spread by respiratory droplets and direct contact (when bodily fluids have touched another person's eyes, nose, or mouth, or an open cut, wound, or abrasion). It should be noted that the SARS-COV-2 virus has been found to be viable on plastic and stainless-steel surfaces for up to 72 hours, whereas on copper and cardboard it is viable for up to 24 hours [4].

Pregnancy is a physiological state that predisposes women to viral respiratory infection. Due to the physiological changes in the immune and cardiopulmonary systems, pregnant women are more likely to develop severe illness after infection with respiratory viruses. In 2009, pregnant women accounted for $1 \%$ of patients infected with influenza A subtype H1N1 virus, but they accounted for $5 \%$ of all H1N1-related deaths.

The clinical presentation of the COVID-19 with pregnancy is same as non-pregnant patient ranging from asymptomatic, mild, flu like illness, severely ill patient with respiratory distress, respiratory failure, organs failure and death.

The investigations of swabs, all blood, serology tests have nothing specific for pregnancy so far. Vital signs monitoring and clinical observation with suppurative measures are the hallmarks in management. Deterioration especially with other comorbidities like obesity and others systemic diseases is expected to be rapid in pregnancy patient.

Regarding vertical transmission (transmission from woman to her baby antenatally or intrapartum), emerging evidence now suggests that vertical transmission is probable, although the proportion of pregnancies affected and the significance to the neonate has yet to be determined. Two reports have published evidence of IgM for SARS-COV-2 in neonatal serum at birth [5] [6]. Since IgM does not cross the placenta, this is likely to represent a neonatal immune response to in utero infection. Previous case reports from China suggested that there was no evidence for this and amniotic fluid, cord blood, neonatal throat swabs, placenta swabs, genital fluid and breastmilk samples from COVID-19 infected mothers have so far all tested negative for the virus [7] [8].

Still the question is raised if SARS-CoV-2 can live in all body fluids with barriers: amniotic fluid and cerebrospinal fluids?

The evidence available nowadays is based on small numbers of reported cases. The situation may change in soon coming future so continue to monitor outcomes of affected women and their babies through careful follow up and col- 
lected analyzed data will be included in future for more evidence-based guidance.

There are currently no data suggesting an increased risk of miscarriage or early pregnancy loss in relation to COVID-19. The data is not enough to prove a relationship between infection and increased risk of miscarriage or second trimester loss as there is no evidence currently that the virus is teratogenic.

The Question of how this infection affecting the pregnancy and its outcome not only for the short term, also for the long term and the baby development, millstones and inelegance functions in future. Is it like rubella bring new fetal anomalies syndrome? Is it like others infection its infection give complications varies from gestational age to another? Still looking for further research and clinical observation the saved outlive group. The real virus activity for crossing the placenta and affection on the new growing embryo and fetal development along with function of the developed and aggression of the infection is not yet clear because the disease is just flared and not yet understood in all views.

Due to the limited data on COVID-19, these recommendations are largely based on infection prevention and control considerations for others respiratory viruses such as influenza, SARS-COV and MERS-COV and are intentionally cautious as experts learn more about this new virus [9].

Considering the limited data, new-borns for COVID-19 positive mothers should be monitored carefully for evidence of transmission.

The lack of evidence related to vertical transmission of COVID-19 virus from the mother to her foetus in the first trimester is a limiting factor in determining the risks of teratogenicity related to the virus itself. Most severe cases of COVID 19 were reported in the third trimester [10].

There are case reports of preterm birth in women with COVID-19, but it is unclear whether this was iatrogenic in every case, or whether some were spontaneous. Iatrogenic birth was predominantly for maternal indications related to the viral infection, although there was evidence of fetal compromise and pre-labour preterm rupture of the membranes in at least one report [9].

The decision of delivery should be taken after multidisciplinary team meeting as case-based decision with respect to the COVID-19 clinical presentations, gestational age and obstetric indication. Choosing the most suiting method of delivery either vaginal delivery which is highly recommended or Caesarean section which is not indicated by COVID-19 per say.

Delivery of serious ill mother special at third trimester helps in maternal suppurative management in assisted ventilation and resuscitation.

Following all precaution and general advice for all population in restrict way will minimize the infection transmission regarding:

1) Personal behaviour and self-protection.

2) Social distance.

3) Stay home policy.

The authors here advice special considerations:

1) No need to attend the routine antenatal clinic. 
2) High risk pregnancy only should attend the ANC with full precaution.

3) Any further consultation if needed better if possible, on phone.

4) Attendance to the emergency in case of genuine indication.

5) No need for any gynaecological intervention or activities unless in case of emergency and should be determine by high technical governance, these case like miscarriage, ectopic pregnancy, scar pregnancy and in all should be managed in shorter time and minimized their hospital stay.

The hallmark of treatment of patients with COVID-19 infection is supportive care. Yet there is no specific treatment so far of concern in pregnancy. Both chloroquine and hydroxychloroquine have been reported to inhibit SARS-COV-2 in vitro, although hydroxychloroquine appears to have more potent antiviral activity [11].

Use of chloroquine is included in treatment guidelines from China's National Health Commission and was reportedly associated with reduced progression of disease and decreased duration of symptoms [12] [13].

Research is running everywhere looking step ahead for actual treatment and vaccination.

Isolation, bereavement, financial difficulties, insecurity and inability to access support systems are all widely recognised risk factors for mental ill-health. The coronavirus epidemic also increases the risk of domestic violence [14].

Some of the disease facts still not yet discovered but considering COVID-19 as serious viral infection keeps the victims and their families under high psychological stress as well the medical staff and all heroes of front lines involving in fighting against COVID-19. It's now well-known newly emerge cause and major risk factor increases the maternal morbidity and mortality.

The authors expecting many psychiatric/psychological issues that may be seen post exposure and after this pandemic control. Women are more liable to emotion stress as being affected or affected by someone affection, so post-traumatic stress and psycho-emotion stress will be the main commonly seen disorders.

Hospitals message to the clients that all elective interventions are postponed. Staff should take care Personal Protective Equipment (PPE) in COVID-19 case management, although its expensive but protection of the frontlines is core in COVID-19 crisis it minimize the stress of getting the infection and with respect of the cost but life of the patient and our staff are counts.

Fighting COVID-19 in Low resources countries at this stage may not differ from the same fighting in developed countries bearing in mind the accessibility of certain areas, whether constrain, local believes in traditional solutions, continuation of the supply for consumables, maintenance of the available resource, support with the needed setup, challenging facing the lockdown and governmental support for financial and daily life needs. All these bring more burden on weak and growing health system that may lasts long after with the poverty and limited resources we expect more burden and failure in health care specially for women and children who are more vulnerable for diseases.

We do recommend after confirmed diagnosis to start the management $b$ the 
supportive therapy, antiviral therapy, antibacterial therapy and introduction of corticosteroids although still is debatable. Timing and mode delivery is crucial along with care of the newborn.

The planners and health strategies care will look after in these constrains as COVID-19 long term health economics; health care system challenges that be start dilemma in viscous cycle manner. Support and collaboration between governments, non-governmental organizations and all stakeholders in health are highly recommended in post COVID-19 pandemic period for all sectors of health.

We do recommend after confirmed diagnosis to start the management by the supportive therapy, antiviral therapy, antibacterial therapy and introduction of corticosteroids although still is debatable. Timing and mode delivery are crucial along with care of the new born.

COVID-19 is spreads around the world; proactive preparation and plan should take place for clinical management and patient support while all adequate protection for healthcare professionals will be offered.

A multi-disciplinary team approach should be adopted in managing these patients as it allows to effectively share the expertise as well as responsibility and treat our patients with dignity and compassion. Clinical recommendations for managing COVID-19 infection in pregnancy should be based on data from the current epidemic rather than drawing on limited experience from previous outbreaks of different types of corona viruses, as their epidemiology, clinical course and response to treatment may differ. Guidelines will evolve as more data become available and experience is gathered. Therefore, complete data on all pregnancies affected by COVID-19 should be collected and made publicly available. Sharing data, knowledge and expertise, and helping countries with poor resources and weaker healthcare systems are important in this respect [15].

\section{Conflicts of Interest}

No conflict of interest between the authors.

\section{References}

[1] Corman, V.M., Muth, D., Niemeyer, D., et al. (2018) Hosts and Sources of Endemic Human Coronaviruses. Advances in Virus Research, 100, 163-188. https://doi.org/10.1016/bs.aivir.2018.01.001

[2] Cui, J., Li, F. and Shi, Z.L. (2019) Origin and Evolution of Pathogenic Coronaviruses. Nature Reviews Microbiology, 17, 181-192. https://doi.org/10.1038/s41579-018-0118-9

[3] Monchatre-Leroyz, E., Boue, F., Boucher, J.M., et al. (2017) Identification of Alpha and Beta Coronavirus in Wildlife Species in France: Bats, Rodents, Rabbits, and Hedgehogs. Viruses, 9, 364. https://doi.org/10.3390/v9120364

[4] Royal College of Obstetrics and Gynaecology 2020.

[5] Dong, L., Tian, J., He, S., et al. (2020) Possible Vertical Transmission of SARS-CoV-2 from an Infected Mother to Her Newborn. JAMA. 
https://doi.org/10.1001/jama.2020.4621

[6] Zeng, H., Xu, C., Fan, J., et al. (2020) Antibodies in Infants Born to Mothers with COVID-19 Pneumonia. JAMA. https://doi.org/10.1001/jama.2020.4861

[7] Chen, H., Guo, J., Wang, C., et al. (2020) Clinical characteristics and Intrauterine Vertical Transmission Potential of COVID-19 Infection in Nine Pregnant Women: A Retrospective Review of Medical Records. The Lancet, 395, 809-815 https://doi.org/10.1016/S0140-6736(20)30360-3

[8] Zhu, H., Wang, L., Fang, C., et al. (2020) Clinical Analysis of 10 Neonates Born to Mothers with 2019-nCoV Pneumonia. Translational Pediatrics, 9, 51-60. https://doi.org/10.21037/tp.2020.02.06

[9] Di Mascio, D., Khalil, A., Saccone, G., et al. (2020) Outcome of Coronavirus Spectrum Infections (SARS, MERS, COVID 1-19) during Pregnancy: A Systematic Review and Meta-Analysis. American Journal of Obstetrics \& Gynecology MFM, In Press. https://doi.org/10.1016/j.ajogmf.2020.100107

[10] Clinical Characteristics and Intrauterine Vertical Transmission Potential of COVID-19 Infection in Nine Pregnant Women: A Retrospective Review of Medical Records. The Lancet, 395, 809-815. https://doi.org/10.1016/S0140-6736(20)30360-3

[11] Yao, X., Ye, F., Zhang, M., et al. (2020) In Vitro Antiviral Activity and Projection of Optimized Dosing Design of Hydroxychloroquine for the Treatment of Severe Acute Respiratory Syndrome Coronavirus 2 (SARS-CoV-2). Clinical Infectious Diseases, ciaa237. https://doi.org/10.1093/cid/ciaa237

[12] Gao, J., Tian, Z. and Yang, X. (2020) Breakthrough: Chloroquine Phosphate Has Shown Apparent Efficacy in Treatment of COVID-19 Associated Pneumonia in Clinical Studies. BioScience Trends, 14, 72.

[13] Colson, P., Rolain, J.M., Lagier, J.C., et al. (2020) Chloroquine and Hydroxychloroquine as Available Weapons to Fight COVID-19. The International Journal of Antimicrobial Agents, 55, 105932.

https://doi.org/10.1016/j.ijantimicag.2020.105932

[14] Fraser, E. (2020) Impact of COVID-19 Pandemic on Violence against Women and Girls. In: Development UAftDoI,ed.,.

[15] Liang, H. and Acharya, G. (2020) Novel Corona Virus Disease (COVID-19) in Pregnancy: What Clinical Recommendations to Follow? Acta Obstetricia et Gynecologica Scandinavica, 99, 439-442. https://doi.org/10.1111/aogs.13836 